\title{
Antley-Bixler syndrome arising from compound heterozygotes in the P450 oxidoreductase gene: a case report
}

\author{
Haibo Li ${ }^{1}$, Aman $\mathrm{Zhao}^{2}, \mathrm{Min}_{\mathrm{Xie}}{ }^{1}$, Linqi Chen ${ }^{2}$, Haiying Wu ${ }^{2}$, Yiping Shen ${ }^{3,4}$, Hongying Wang ${ }^{2,5}$ \\ ${ }^{1}$ The Central Laboratory of Birth Defects Prevention and Control, Ningbo Women and Children's Hospital, Ningbo, China; ${ }^{2}$ Department of \\ Clinical Laboratory, Children's Hospital of Soochow University, Suzhou, China; ${ }^{3}$ Division of Genetics and Genomics, Department of Medicine, \\ Boston Children's Hospital, Boston, MA, USA; ${ }^{4}$ Department of Neurology, Harvard Medical School, Boston, MA, USA; ${ }^{5}$ Department of Clinical \\ Laboratory, Children's Hospital of Soochow University (Wujiang District), Suzhou, China \\ Correspondence to: Hongying Wang. Clinical Laboratory, Children's Hospital of Soochow University (Wujiang District), Suzhou, China; Department \\ of Clinical Laboratory, Children's Hospital of Soochow University, Suzhou, China. Email: why923811@sina.com; Haibo Li. The Central Laboratory \\ of Birth Defects Prevention and Control, Ningbo Women and Children's Hospital, Ningbo, China. Email: lihaibo-775@163.com.
}

\begin{abstract}
Antley-Bixler syndrome (ABS) arising from P450 oxidoreductase deficiency (PORD) is a rare, distinct craniosynostosis syndrome, accompanied by ambiguous genitalia and impaired steroidogenesis. It is reported that this disorder is caused by mutations in the P450 oxidoreductase (POR; OMIM \#124015) gene via autosomal recessive inheritance. In this study, we performed a molecular analysis to verify the genetic etiology of ABS in an infant. Initially, medical exome sequencing was applied using the parents' peripheral blood genome DNA. Next, bidirectional Sanger sequencing and quantitative real-time PCR (qRT-PCR) were conducted to confirm the sequencing results. The infant was diagnosed as ABS at birth, with typical midface hypoplasia, craniosynostosis, femoral bowing, radio-ulnar synostosis, and genital anomalies. She died two months later due to severe pneumonia and congenital heart disease. The medical exome sequencing and Sanger sequencing revealed the missense mutation c.1370G>A (p.R457H) in exon 12 of $P O R$ was inherited from the father. In addition, the qRT-PCR analysis verified an exon 5 microdeletion in the $P O R$ gene of the infant and her mother. While p.R457H is a well-known pathogenic mutation, the POR exon 5 deletion is absent from the public databases. However, it is classified as pathogenic according to the American College of Medical Genetics and Genomics (ACMG) guidelines based on the evidence of PVS1, PM2, and PM3. In conclusion, this infant with ABS carried compound heterozygotic mutations in the POR gene; one was a paternal missense mutation, and the other was a maternal novel microdeletion. The mutations were inherited from the paternal grandfather and maternal grandfather, respectively. This detailed case report enriches our knowledge of the POR mutation spectrum and ABS pathogenesis.
\end{abstract}

Keywords: Case report; Antley-Bixler syndrome (ABS); P450 oxidoreductase (POR); exome sequencing; ambiguous genitalia

Submitted Aug 23, 2021. Accepted for publication Dec 06, 2021.

doi: $10.21037 / \mathrm{tp}-21-499$

View this article at: https://dx.doi.org/10.21037/tp-21-499

\section{Introduction}

Antley-Bixler syndrome (ABS), first described by Antley and Bixler in 1975, is a rare skeletal malformation syndrome (1). The most characteristic clinical features of ABS patients include midface hypoplasia, craniosynostosis, radioulnar synostosis, choanal atresia or stenosis, femoral bowing and fractures, joint contractures, and arachnodactyly (2). Studies have shown that ventriculoperitoneal shunts are clinically required for treating craniosynostosis-induced hydrocephalus, and tracheotomy is usually provided to ABS patients with extreme midface hypoplasia and respiratory difficulties. In the neonatal period, the mortality rate of ABS is reportedly around $80 \%$ and is primarily caused by 
airway compromise (3).

The pathogenesis of ABS is complicated and often influenced by genetic heterogeneity, including mutations in fibroblast growth factor receptor 2 (FGFR2) mutation and cytochrome P450 oxidoreductase (POR) (4-6). FGFR2 related ABS is autosomal dominant and $P O R$ related $\mathrm{ABS}$ is autosomal recessive. It is noteworthy that cases of ABS with the POR mutation manifest genital anomalies and disordered steroidogenesis in addition to skeletal malformation, while cases with FGFR2 mutations are absent of genital anomalies. Male micropenis, hypospadias, and cryptorchidism, as well as female vaginal atresia, labia majora hypoplasia, labia minora fusion, and clitoromegaly, are characterized as $P O R$ mutation-induced genital abnormalities $(7,8)$.

The human POR gene is located on the long arm of chromosome 7 ( $7 \mathrm{q} 11.2)$. Electrons are transferred by this gene from reduced nicotinamide adenine dinucleotide phosphate (NADPH) to all microsomal P450 enzymes, which are essential for steroid hormone synthesis and drug and toxin metabolism (9). Therefore, mutations in POR could result in P450 enzyme deficiency. Indeed, deficiencies in the steroidogenic P450 enzymes including CYP17A1, CYP21A2, and CYP51A1 are most commonly associated with $\operatorname{ABS}(2,7,10)$. To date, a number of mutations, including missense, nonsense, splice site, and frameshift, as well as microdeletions or microduplications have been reported in the POR gene region (11-14). Previous studies have shown that the A287P missense POR mutation resulted in a $40 \%$ residual $P O R$ activity and has been proved to be the most frequent mutation in Caucasian patients, whereas $\mathrm{R} 457 \mathrm{H}$ occurred in over $70 \%$ of Japanese patients and led to a $3 \%$ residual POR activity (15-18). A number of $P O R$ related ABS cases have been reported previously $(3-5,19,20)$. However, only a small proportion of them are based on Chinese patients and few of them screened the mutations for the family members (including parents and grandparents) of the patients.

This study reports on a 46, XX newborn (a newborn with a 46, XX karyotype) with skeletal malformations and genital abnormalities, clinically diagnosed as ABS. This newborn had compound heterozygotes in the POR gene. Genetic analysis confirmed that the mutations were a paternal missense mutation and a maternal small deletion inherited from the paternal grandfather and maternal grandfather, respectively. By providing a detailed description of this rare $\mathrm{ABS}$ case from a genetic perspective, we aim to contribute to the existing body of knowledge regarding
ABS pathogenesis and enrich the information available for ABS genetic counseling. We present the following article in accordance with the CARE reporting checklist (available at https://dx.doi.org/10.21037/tp-21-499).

\section{Case presentation}

\section{Diagnostic criteria}

The observed characteristic clinical phenotypes of skeletal and craniofacial malformation (including craniosynostosis, low set ears, midface hypoplasia, cloverleaf skull, and pearshaped nose) and genital abnormalities were used as a preliminary diagnosis of ABS. A subsequent radiography scan was used to confirm the diagnosis. The research was carried out in compliance with the Declaration of Helsinki (as revised in 2013) and written informed consent was obtained from the patient's guardian for publication of this case report and accompanying images. A copy of the written consent is available for review by the editorial office of this journal.

\section{Peripheral blood collection}

The Institutional Ethics Committee of Children's Hospital of Soochow University approved this study. Peripheral blood was then collected for genomic DNA extraction.

\section{Medical exome sequencing}

Genomic DNA was extracted from the peripheral blood using a QIAamp DNA Mini Kit (Qiagen, Germany) according to the manufacturer's instructions. The Illumina HiSeq 2500 System (Illumina, USA) and the Agilent SureSelect XT Inherited Disease Panel (Agilent Technologies, USA) containing 2,742 genes were used for the medical exome sequencing. Candidate variants were screened by Ingenuity Variant Analysis (Ingenuity Systems, USA), and subsequent data analysis was performed with NextGENe (SoftGenetics LLC, USA) (21).

\section{Bidirectional Sanger sequencing}

The candidate causal variations identified by medical exome sequencing were further confirmed by Sanger sequencing. Faststart Taq DNA polymerase (Roche, Switzerland) was used to amplify the exon 12 sequence of the POR gene (including the exon-intron boundaries) by polymerase chain 

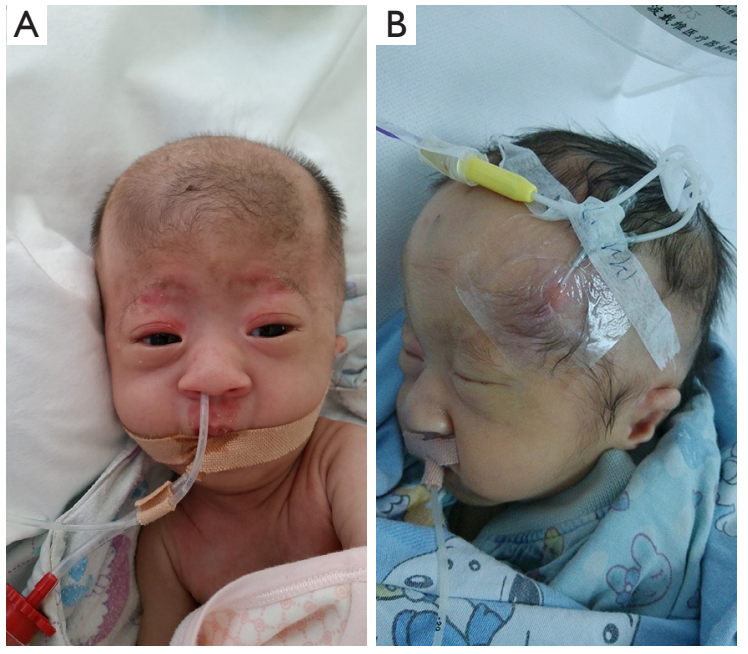

Figure 1 Photograph of the patient showing craniosynostosis, cloverleaf skull, midface hypoplasia, low set ears, and a pear-shaped nose. These images are published with the consent from patients' parents.
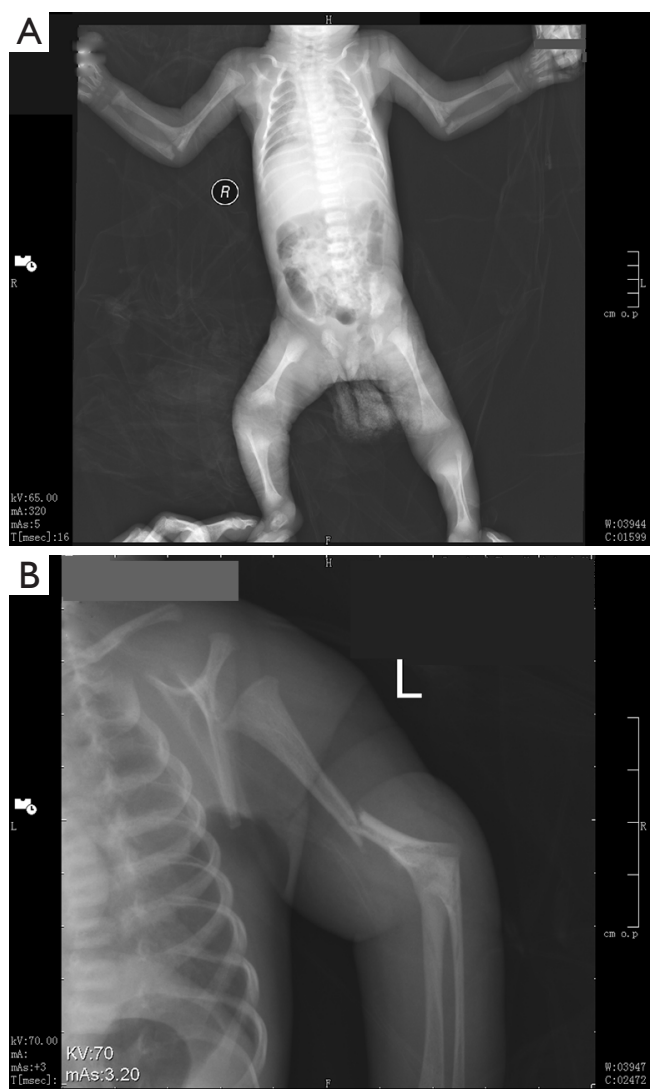

Figure 2 Bilateral femora deformation, radio-ulnar synostosis, femoral bowing and fractures, joint contractures, which were combined as limb malformations showing in this radiograph. reaction (PCR). The primer sequences designed for POR gene amplification are shown in Table S1. An ABI 3130 Genetic Analyzer was then used to purify and sequence the amplified DNA fragments in both directions. Finally, we compared the sequencing data to the reference sequence of POR (NM_000941.2) from the National Center for Biotechnology Information (NCBI) database.

\section{Quantitative real-time PCR (qRT-PCR) detection}

SYBR Premix Ex Taq (Takara, Dalian China) was used to perform the qRT-PCR analysis. The results were then normalized according to the expression of glyceraldehyde 3-phosphate dehydrogenase (GAPDH). Table S1 shows the specific primers used in this study. An ABI 7500 real-time PCR system (Applied Biosystems, USA) was used for the qRT-PCR assays. The results were then converted to fold changes after being analyzed and expressed relative to the threshold cycle (CT) values.

\section{Clinical features}

This 46, XX newborn was referred to our neonatal ward soon after birth due to bronchopneumonia and multiple malformations. A preliminary diagnosis of ABS was made based on the observed characteristic clinical phenotypes of skeletal malformation and genital abnormalities. The craniofacial malformations are shown in Figure 1 and includ craniosynostosis, low set ears, midface hypoplasia, cloverleaf skull, and a pear-shaped nose. A subsequent radiography scan revealed several limb malformations, including bilateral femora deformation, femoral bowing and fractures, radio-ulnar synostosis, and joint contractures (Figure 2). Characteristic genital abnormalities, including labia majora hypoplasia, labia minora fusion, clitoromegaly, and vaginal atresia were also observed in this ABS patient (Figures 3,4). This suffering infant died two months later due to severe pneumonia, dyspnea, and congenital heart disease. There was no family history of these disorders, but the two previous pregnancies of the proband's mother were both stillborn.

\section{Genetic analysis}

The medical exome sequencing of the proband's parents identified a heterozygous mutation c.1370G >A (p.R457H) in exon 12 of the father's $P O R$ gene and suspected deletions in exons 4 and 5 of the mother's $P O R$ gene. The c.1370G $>\mathrm{A}$ 


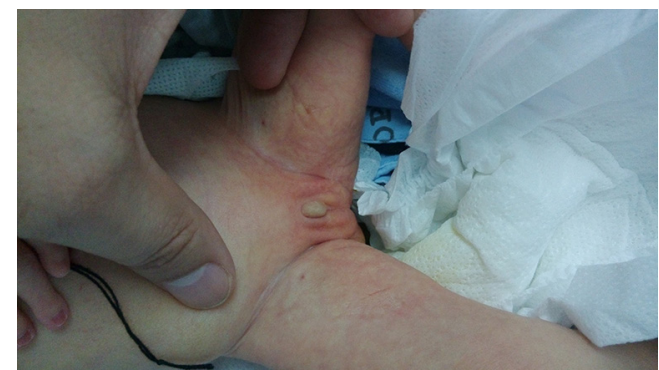

Figure 3 Photograph of the patient showing Labia majora hypoplasia, labia minora fusion and clitoromegaly.

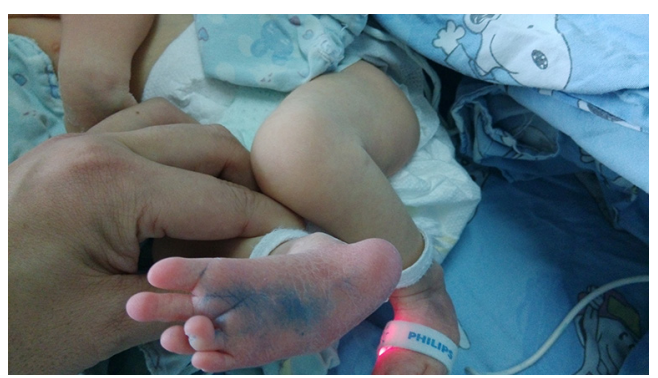

Figure 4 Photograph showing abnormal bone of Antley-Bixler syndrome (ABS) patient caused by $\mathrm{P} 450$ oxidoreductase deficiency (PORD).

(p. $\mathrm{R} 457 \mathrm{H})$ mutation has been reported to occur in over $70 \%$ of Japanese patients and is recognized as a pathogenic mutation by the Human Gene Mutation Database (HGMD) (5).

Subsequently, bidirectional Sanger sequencing was used to verify the NM_000941.3: c.1370G>A (p.R457H) mutation in all available family members (Figure $5 A$ ). The results showed that the ABS infant carried a c.1370G $>A$ (p. R457H) mutation in $P O R$ that was inherited from her father and paternal grandfather (Figure 5B). Furthermore, the results of the qRT-PCR assay revealed that this ABS patient had a microdeletion in exon 5 of the $P O R$ gene (Figure $5 C$ ) that was inherited from her mother and maternal grandfather. The POR exon 5 deletion is absent from the public databases but is classified as pathogenic according to the American College of Medical Genetics and Genomics (ACMG) guidelines based on the evidence of PVS1, PM2, and PM3 (22).

In summary, compound heterozygotic mutations in the $P O R$ gene were identified in this 46, XX ABS-diagnosed infant. The mutations compromised a paternal missense mutation and a maternal small deletion, inherited from the paternal grandfather and maternal grandfather, respectively.

\section{Discussion}

Antley-Bixler syndrome (ABS) as a rare disorder has no cure at the current stage. Although a number of treatments can be performed to manage specific symptoms seen in each individual, they are used as supportive. The prognosis of ABS depends on the severity of malformation. Its mortality rate is around $80 \%$ in the first months of life (23), however, for individuals with mild malformation, their prognosis improves with age. Studies have shown that ABS phenotypes are caused by two different genetic etiologies. One ABS type includes genital anomalies and disordered steroidogenesis (ABS1; OMIM \#201750) and is caused by mutations in the genomic region encoding cytochrome POR (OMIM \#124015). The other form of ABS has normal genitalia and steroidogenesis (ABS2; OMIM \#207410) and is caused by mutations in the FGFR2 gene (OMIM \#176943) (3,8,24-26). ABS caused by $\mathrm{P} 450$ oxidoreductase deficiency (PORD) is a rare disease and can be easily misdiagnosed in mild cases. This disease should be considered in patients with sexual dysfunction, especially when specific skeletal deformities are also present. Craniosynostosis, femoral bowing, radio-ulnar synostosis, midface hypoplasia, and fractures have been recognized as the primary clinical features, and mutation analysis of the FGFR2 and POR genes is necessary to assist in an ABS diagnosis. Autosomal recessive inheritance of the POR mutation should be considered to confirm the diagnosis when skull deformity, limb skeletal malformations, and genital abnormalities occur together.

PORD resulting from mutations in the POR gene was first identified in patients with ambiguous genitalia, defects in steroidogenic cytochrome $\mathrm{P} 450$ enzymes, and ABS-like skeletal malformations $(8,27)$. Currently, over 140 cases of PORD have been described worldwide, and around $85 \%$ of patients with POR enzyme deficiency have ABS-like skeletal deformities (https://www.ncbi.nlm.nih.gov/books/ NBK1419/\#). The majority of reported PORD cases are of Japanese or European descent, and more than 90 mutations in the POR gene have been identified (http://www.hgmd. cf.ac.uk/ac/index.php) $(11,13,14,20,25,28-31)$. There have been few PORD case cohorts reported in China (4). Hence, we performed a genotype-phenotype correlational analysis on 21 Chinese patients identified with POR gene mutations, based on the literature published in Chinese or English (Table 1) (1-4,7,13,32-38).

According to the summarized information, PORD in 
A

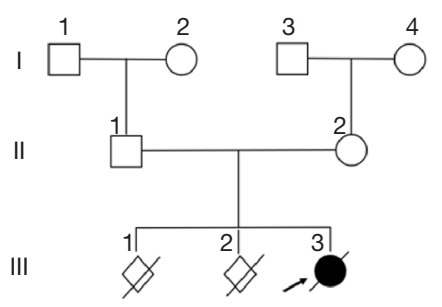

C

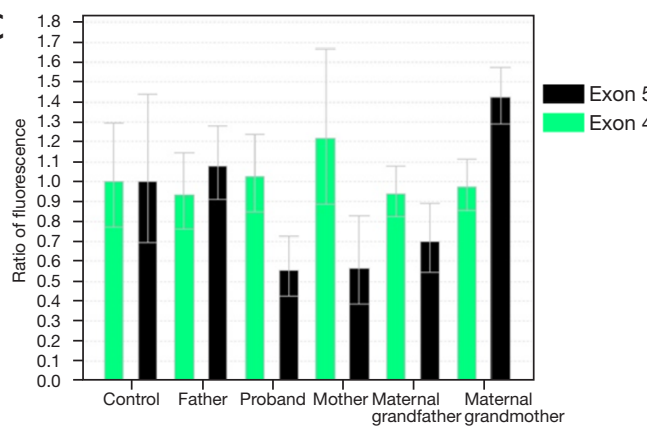

B

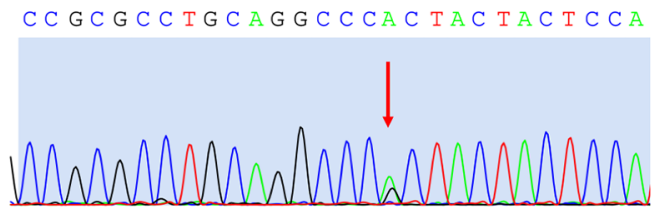

C C GCGCCIGCAGGCCCRCIACTACT C CA

Father

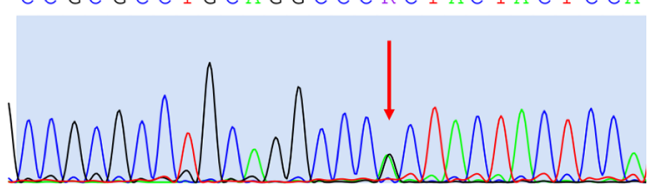

C C G C G C C T G C A G C C C G C TACTACTC CA

Mother

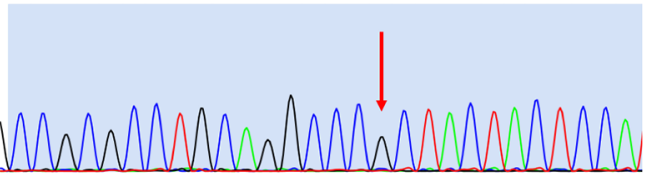

c. $1370 . \mathrm{G}>\mathrm{A}(\mathrm{p} . \mathrm{R} 457 \mathrm{H})$

heterozygous

c. $1370 . \mathrm{G}>\mathrm{A}(\mathrm{p} . \mathrm{R} 457 \mathrm{H})$

heterozygous

Wild type

Figure 5 Genetic testing results of the patient and her parents. (A) Heredity map of proband family. (B) Sanger sequencing result indicates proband carried a c.1370G>A (p.R457H) mutation in P450 oxidoreductase (POR), which was inherited from her father. (C) RT-PCR assay revealed proband had exon 5 deletions of $P O R$ gene, which inherited from her mother and maternal grandfather.

Chinese patients has a wide range of clinical manifestations. These manifestations include menstrual disorders, skeletal malformations, severe hermaphroditism, and occasionally death. To date, 13 POR mutations have been reported in 21 Chinese patients, including eight missense, two nonsense, two frameshift, and one splice site. Remarkably, the most frequent mutation in the Chinese population is $\mathrm{p} . \mathrm{R} 457 \mathrm{H}$ $(18 / 21,85.7 \%)$, which is the same founder mutation identified in Japanese patients $(11,15,20)$. Five cases in the Chinese cohort carried homozygous p.R $457 \mathrm{H}$, and the P399_E401 deletion reported first in Turkish families was found in one Chinese case (39). To date, p.A287P, which has a high prevalence in Caucasian populations, has not been detected in Chinese PORD patients $(13,14,25)$.

Since the effect of variants in the $P O R$ gene may vary among different redox partners and substrates, the broad phenotypic spectrum of PORD may be caused by different POR-dependent P450 enzymatic deficiencies resulting from various $P O R$ variants $(10,16,40)$. Researchers have shown that patients harboring a null mutation in one allele display more severe skeletal malformations than those with homozygotes for p.R457H or p.A287P (11,14,25). Similarly, in the Chinese cohort, subjects (P1, P9, P10, P13, and P14) harboring compound heterozygosity for $\mathrm{R} 457 \mathrm{H}$ and one null mutation showed more severe craniofacial and skeletal abnormalities than the cases with homozygosity for $\mathrm{R} 457 \mathrm{H}$
(P2, P3, P11, P16, and P19) (Table 1). Notably, our patient in this study carried compound heterozygosity of a p.R457H missense variant and a novel exon 5 deletion in the POR gene, manifesting as severe ABS malformations, cardiac defects, and early demise in infancy. In another study, severe dysmorphic and skeletal malformations were found in three sibling fetuses with compound heterozygosity in the POR gene; one mutation was a copy number deletion, and the other was a p.A287P missense mutation (41).

In this case study, exome sequencing revealed compound heterozygosity of a paternal missense mutation p.R $457 \mathrm{H}$ and a maternal novel exon 5 deletions in the POR gene of a 46, XX karyotype newborn. This finding highlights the need for $P O R$ gene copy number analysis for suspected ABS patients harboring only one or no pathogenic variant detected by sequence analysis $(19,26,42)$. However, confirmation from other studies based on more samples is required. Meanwhile, further investigation of the biological mechanism of the POR gene is also necessary for understanding its role in causing ABS.

In conclusion, the causative variants identified in this study have broadened our knowledge of the POR gene mutation spectrum. The accurate genetic diagnosis was valuable for prognosis in this case. Meanwhile, an accurate genetic diagnosis would also be highly valuable for genetic counseling of at-risk couples. It would provide them with 


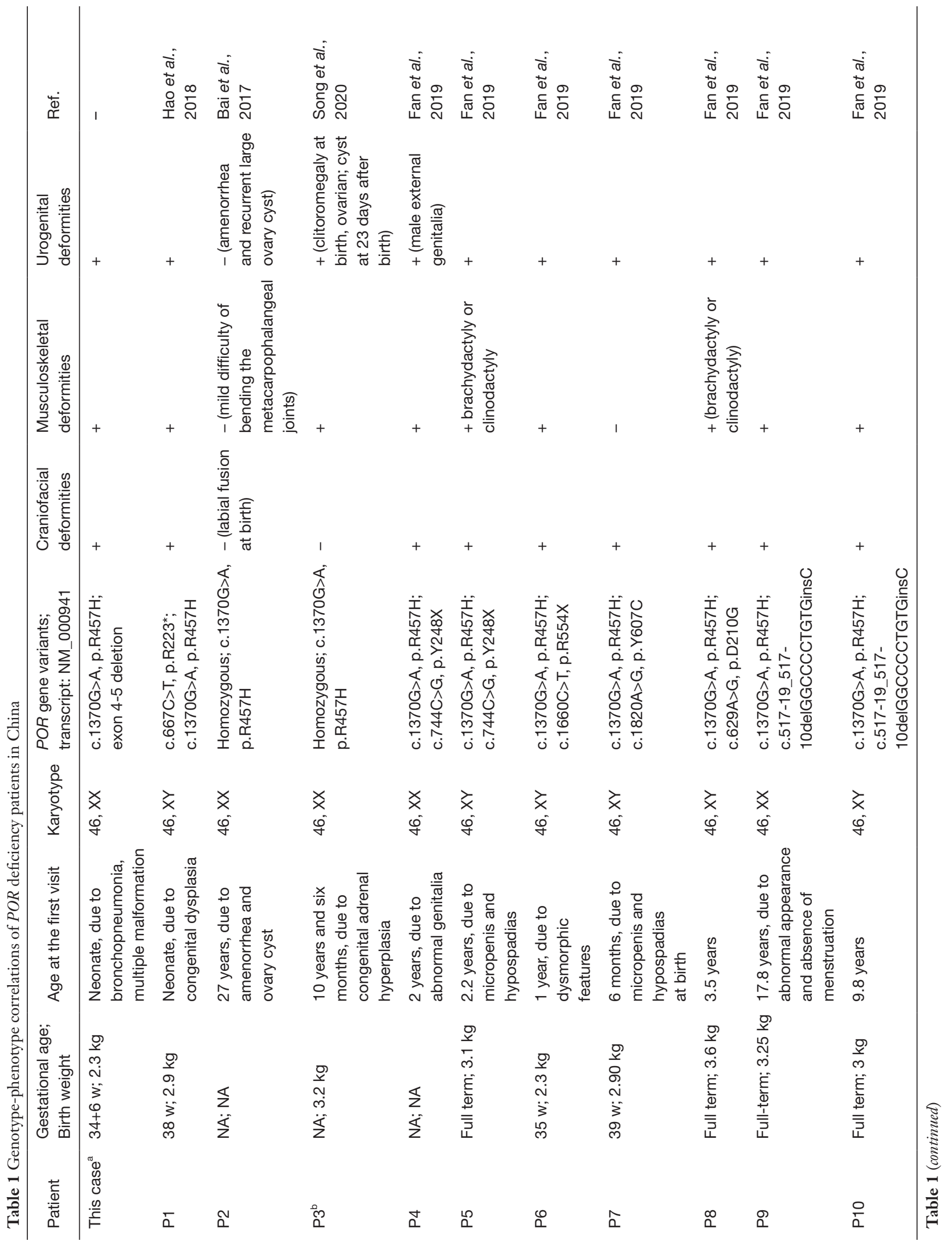




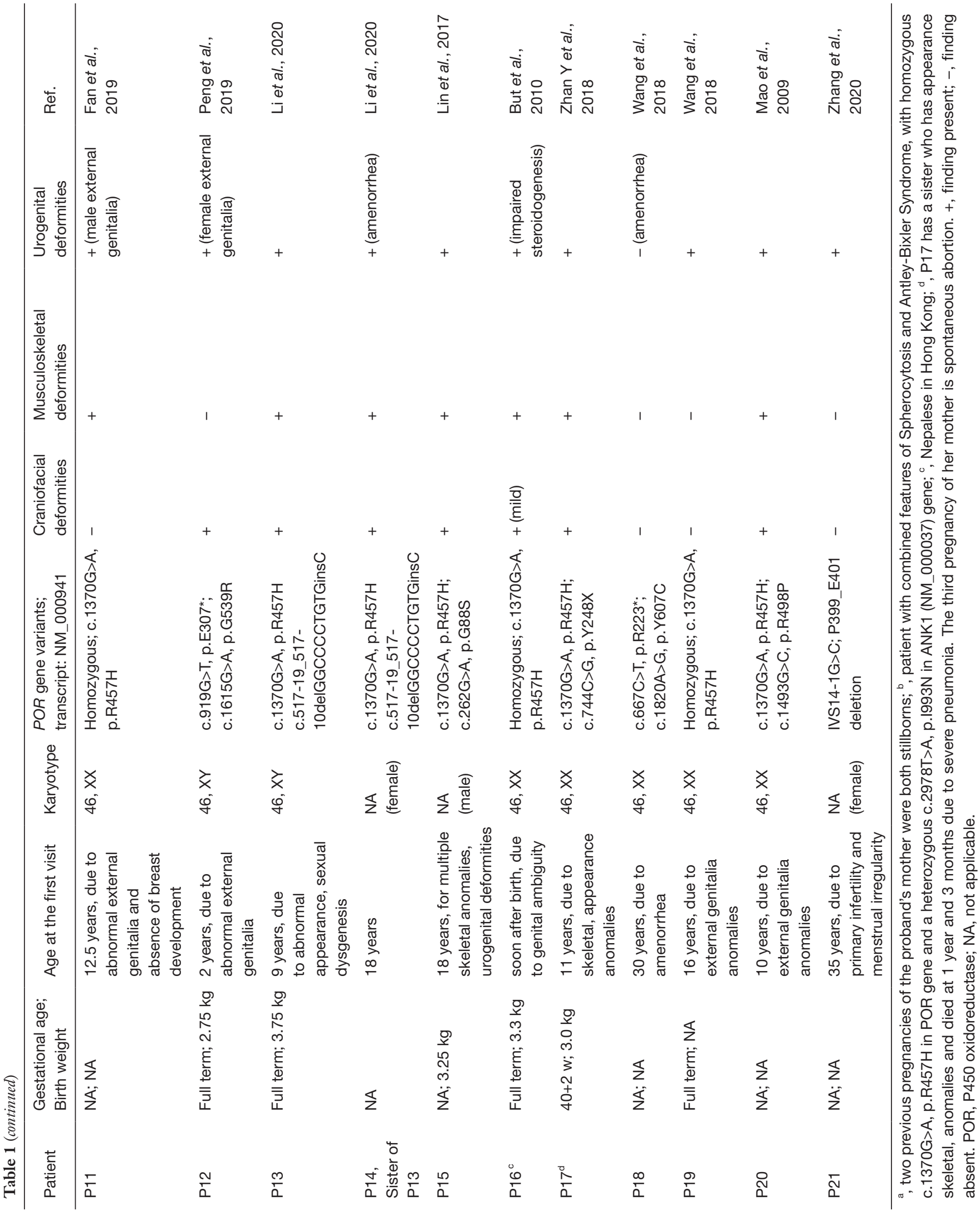


the opportunity to undergo either a prenatal diagnosis or IVF followed by a preimplantation genetic diagnosis.

\section{Acknowledgments}

We would like to thank the proband's family for their cooperation and interest to participate in this study.

Funding: This study was supported by the Social Development Public Welfare Foundation of Ningbo (Grant No. 202002N3150, 2019C50070), First Municipal Medical and Health Brand Foundation of Ningbo (Grant No. PPXK2018-06), The Medical and Health Project of Zhejiang Province (Grant No. 2020KY890), and National Key R\&D Projects (Grant No. 2017YFC1001703).

\section{Footnote}

Reporting Checklist: The authors have completed the CARE reporting checklist. Available at https://dx.doi. org/10.21037/tp-21-499

Conflicts of Interest: All authors have completed the ICMJE uniform disclosure form (available at https://dx.doi. org/10.21037/tp-21-499). The authors have no conflicts of interest to declare.

Ethical Statement: The authors are accountable for all aspects of the work in ensuring that questions related to the accuracy or integrity of any part of the work are appropriately investigated and resolved. The Institutional Ethics Committee of Children's Hospital of Soochow University approved this study. All procedures performed in studies involving human participants were in accordance with the ethical standards of the institutional and/or national research committee(s) and with the Helsinki Declaration (as revised in 2013). Written informed consent was obtained from the patient's guardian for publication of this case report and accompanying images. A copy of the written consent is available for review by the editorial office of this journal.

Open Access Statement: This is an Open Access article distributed in accordance with the Creative Commons Attribution-NonCommercial-NoDerivs 4.0 International License (CC BY-NC-ND 4.0), which permits the noncommercial replication and distribution of the article with the strict proviso that no changes or edits are made and the original work is properly cited (including links to both the formal publication through the relevant DOI and the license). See: https://creativecommons.org/licenses/by-nc-nd/4.0/.

\section{References}

1. Hao C, Guo J, Guo R, et al. Compound heterozygous variants in POR gene identified by whole-exome sequencing in a Chinese pedigree with cytochrome P450 oxidoreductase deficiency. Pediatr Investig 2018;2:90-5.

2. Bai Y, Li J, Wang X. Cytochrome P450 oxidoreductase deficiency caused by $\mathrm{R} 457 \mathrm{H}$ mutation in POR gene in Chinese: case report and literature review. J Ovarian Res 2017;10:16.

3. Song F, Feng S, Shen X, et al. Next-Generation Sequencing Revealed Disease-Causing Variants in Two Genes in a Patient With Combined Features of Spherocytosis and Antley-Bixler Syndrome With Genital Anomalies and Disordered Steroidogenesis. Front Genet 2020;11:976.

4. Fan L, Ren X, Song Y, et al. Novel phenotypes and genotypes in Antley-Bixler syndrome caused by cytochrome P450 oxidoreductase deficiency: based on the first cohort of Chinese children. Orphanet J Rare Dis 2019;14:299.

5. Flück CE, Tajima T, Pandey AV, et al. Mutant P450 oxidoreductase causes disordered steroidogenesis with and without Antley-Bixler syndrome. Nat Genet 2004;36:228-30.

6. Arlt W, Walker EA, Draper N, et al. Congenital adrenal hyperplasia caused by mutant $\mathrm{P} 450$ oxidoreductase and human androgen synthesis: analytical study. Lancet 2004;363:2128-35.

7. But WM, Lo IF, Shek CC, et al. Ambiguous genitalia, impaired steroidogenesis, and Antley-Bixler syndrome in a patient with P450 oxidoreductase deficiency. Hong Kong Med J 2010;16:59-62.

8. Reardon W, Smith A, Honour JW, et al. Evidence for digenic inheritance in some cases of Antley-Bixler syndrome? J Med Genet 2000;37:26-32.

9. Pandey AV, Sproll P. Pharmacogenomics of human P450 oxidoreductase. Front Pharmacol 2014;5:103.

10. Miller WL. P450 oxidoreductase deficiency: a disorder of steroidogenesis with multiple clinical manifestations. Sci Signal 2012;5:pt11.

11. Fukami M, Nishimura G, Homma K, et al. Cytochrome P450 oxidoreductase deficiency: identification and characterization of biallelic mutations and genotypephenotype correlations in 35 Japanese patients. J Clin 
Endocrinol Metab 2009;94:1723-31.

12. Soneda S, Yazawa T, Fukami M, et al. Proximal promoter of the cytochrome $\mathrm{P} 450$ oxidoreductase gene: identification of microdeletions involving the untranslated exon 1 and critical function of the SP1 binding sites. J Clin Endocrinol Metab 2011;96:E1881-7.

13. Zhang T, Li Z, Ren X, et al. Clinical and genetic analysis of cytochrome $\mathrm{P} 450$ oxidoreductase (POR) deficiency in a female and the analysis of a novel POR intron mutation causing alternative mRNA splicing : Overall analysis of a female with POR deficiency. J Assist Reprod Genet 2020;37:2503-11.

14. Krone N, Reisch N, Idkowiak J, et al. Genotypephenotype analysis in congenital adrenal hyperplasia due to P450 oxidoreductase deficiency. J Clin Endocrinol Metab 2012;97:E257-67.

15. Fukami M, Ogata T. Cytochrome P450 oxidoreductase deficiency: rare congenital disorder leading to skeletal malformations and steroidogenic defects. Pediatr Int 2014;56:805-8.

16. Miller WL, Agrawal V, Sandee D, et al. Consequences of POR mutations and polymorphisms. Mol Cell Endocrinol 2011;336:174-9.

17. Saito Y, Yamamoto N, Katori N, et al. Genetic polymorphisms and haplotypes of por, encoding cytochrome p450 oxidoreductase, in a Japanese population. Drug Metab Pharmacokinet 2011;26:107-16.

18. Dhir V, Ivison HE, Krone N, et al. Differential inhibition of CYP17A1 and CYP21A2 activities by the P450 oxidoreductase mutant A287P. Mol Endocrinol 2007;21:1958-68.

19. Imataka G, Fujisawa M, Kuribayashi R, et al. Low-birthweight infant with Antley-Bixler syndrome-like phenotype caused by POR mutation: a rare case report. Eur Rev Med Pharmacol Sci 2020;24:11998-2000.

20. Fukami M, Horikawa R, Nagai T, et al. Cytochrome P450 oxidoreductase gene mutations and Antley-Bixler syndrome with abnormal genitalia and/or impaired steroidogenesis: molecular and clinical studies in 10 patients. J Clin Endocrinol Metab 2005;90:414-26.

21. Li N, Chang G, Xu Y, et al. Clinical and Molecular Characterization of Patients with Fructose 1,6-Bisphosphatase Deficiency. Int J Mol Sci 2017;18:857.

22. Richards S, Aziz N, Bale S, et al. Standards and guidelines for the interpretation of sequence variants: a joint consensus recommendation of the American College of Medical Genetics and Genomics and the Association for Molecular Pathology. Genet Med 2015;17:405-24.
23. Hosalkar HS, Shah HS, Gujar PS, et al. The Antley-Bixler syndrome: two new cases. J Postgrad Med 2001;47:252-5.

24. McGlaughlin KL, Witherow H, Dunaway DJ, et al. Spectrum of Antley-Bixler syndrome. J Craniofac Surg 2010;21:1560-4.

25. Huang N, Pandey AV, Agrawal V, et al. Diversity and function of mutations in p450 oxidoreductase in patients with Antley-Bixler syndrome and disordered steroidogenesis. Am J Hum Genet 2005;76:729-49.

26. Oh J, Song JS, Park JE, et al. A Case of AntleyBixler Syndrome With a Novel Likely Pathogenic Variant (c.529G>C) in the POR Gene. Ann Lab Med 2017;37:559-62.

27. Tomková M, Marohnic CC, Baxová A, et al. AntleyBixler syndrome or POR deficiency?. Cas Lek Cesk 2008;147:261-5.

28. Adachi M, Tachibana K, Asakura Y, et al. Compound heterozygous mutations of cytochrome $\mathrm{P} 450$ oxidoreductase gene (POR) in two patients with AntleyBixler syndrome. Am J Med Genet A 2004;128A:333-9.

29. Baronio F, Ortolano R, Menabò S, et al. 46,XX DSD due to Androgen Excess in Monogenic Disorders of Steroidogenesis: Genetic, Biochemical, and Clinical Features. Int J Mol Sci 2019;20:4605.

30. Flück CE, Nicolo C, Pandey AV. Clinical, structural and functional implications of mutations and polymorphisms in human NADPH P450 oxidoreductase. Fundam Clin Pharmacol 2007;21:399-410.

31. Sahakitrungruang T, Huang N, Tee MK, et al. Clinical, genetic, and enzymatic characterization of P450 oxidoreductase deficiency in four patients. J Clin Endocrinol Metab 2009;94:4992-5000.

32. Li H, Dong R, Zhang K, et al. [Clinical and genetic analysis of a pedigree affected with cytochrome $\mathrm{P} 450$ oxidoreductase deficiency]. Zhonghua Yi Xue Yi Chuan Xue Za Zhi 2020;37:1005-8.

33. Lin J, Zheng J, Nie M, et al. Approach to the patient with cytochrome P450 oxidoreductase deficiency. Chin J Endocrinol Metab 2017;33:68-71.

34. Mao J, Wu X, Nie M, et al. Clitoral enlargementadolescent agenesis-polycystic ovary-skeletal malformation. Nati Med J China 2009;89:3304-6.

35. Pan P, Zheng L, Chen X, et al. Successful live birth in a Chinese woman with P450 oxidoreductase deficiency through frozen-thawed embryo transfer: a case report with review of the literature. J Ovarian Res 2021;14:22.

36. Peng C, Huang C, Tan H, et al. A case of Antley-Bixler syndrome caused by novel POR mutations. Zhonghua Yi 
Xue Yi Chuan Xue Za Zhi 2019;36:1025-7.

37. Wang W, L. Y, Yang Z, et al. Clinical characteristics and molecular diagnosis in 2 cases of $\mathrm{P} 450$ oxidoreductase deficiency. Chin J Endocrinol Metab 2018;34:926-30.

38. Zhan $Y$, Cheng L, Xiong $M$, et al. Congenital adrenal hyperplasia due to cytochrome $\mathrm{P} 450$ oxidoreductase gene mutation: a case report. Chin J Perinat Med 2018;21:825-9.

39. Flück CE, Mallet D, Hofer G, et al. Deletion of P399_ E401 in NADPH cytochrome P450 oxidoreductase results in partial mixed oxidase deficiency. Biochem Biophys Res Commun 2011;412:572-7.

40. Parween S, Rojas Velazquez MN, Udhane SS, et al. Variability in Loss of Multiple Enzyme Activities Due

Cite this article as: $\mathrm{Li} \mathrm{H}$, Zhao $\mathrm{A}$, Xie $\mathrm{M}$, Chen $\mathrm{L}, \mathrm{Wu}$ H, Shen Y, Wang H. Antley-Bixler syndrome arising from compound heterozygotes in the $\mathrm{P} 450$ oxidoreductase gene: a case report. Transl Pediatr 2021;10(12):3309-3318. doi: $10.21037 /$ tp-21-499 to the Human Genetic Variation P284T Located in the Flexible Hinge Region of NADPH Cytochrome P450 Oxidoreductase. Front Pharmacol 2019;10:1187.

41. Tzetis M, Konstantinidou A, Sofocleous C, et al. Compound heterozygosity of a paternal submicroscopic deletion and a maternal missense mutation in POR gene: Antley-bixler syndrome phenotype in three sibling fetuses. Birth Defects Res A Clin Mol Teratol 2016;106:536-41.

42. Mao J, Nie M, Gao J, et al. P450 Oxidoreductase Deficiency: Clinical Manifestations and Prenatal Diagnosis by Amniocentesis. Basic \& Clinical Medicine 2013;33:24-7.

(English Language Editor: D. Fitzgerald) 
Supplementary

Table S1 List of primer sequences

\begin{tabular}{|c|c|c|c|c|c|}
\hline \multirow{2}{*}{ Gene } & \multirow{2}{*}{ Mutation } & \multicolumn{4}{|c|}{ Primer } \\
\hline & & Sequencing region & Direction & Primer sequence $\left(5^{\prime}-3^{\prime}\right)$ & Size (bp) \\
\hline \multirow[t]{2}{*}{$P O R$} & \multirow{2}{*}{$\begin{array}{l}\text { c. } 1370 G>A \\
\text { (p.R457H) }\end{array}$} & \multirow[t]{2}{*}{ POR-ex12 } & $\mathrm{F}$ & CCTGGACATCACCAACCCG & 19 \\
\hline & & & $\mathrm{R}$ & САCCTGGCACCCAAACCTC & 19 \\
\hline \multirow[t]{6}{*}{$P O R$} & \multirow[t]{6}{*}{ Exon 5 deletion } & \multirow[t]{2}{*}{ POR-ex4 } & $\mathrm{F}$ & TTGGTCAGGCTGGTCTTG & 18 \\
\hline & & & $\mathrm{R}$ & GACCTGCTCCCTGTCCTAC & 19 \\
\hline & & \multirow[t]{2}{*}{ POR-ex5 } & $\mathrm{F}$ & CCCACGACACTCAGACATCC & 20 \\
\hline & & & $\mathrm{R}$ & TCCACTGCCAGCCTCAAC & 18 \\
\hline & & \multirow[t]{2}{*}{ POR-ex4ex5 } & $\mathrm{F}$ & TGCCTTGGTGACCTTTGC & 18 \\
\hline & & & $\mathrm{R}$ & GGGCTGCCTTTCAGATACA & 19 \\
\hline
\end{tabular}

POR, P450 oxidoreductase; F, forward direction; R, reverse direction 